\title{
Gefitinib suppresses cervical cancer progression by inhibiting cell cycle progression and epithelial-mesenchymal transition
}

\author{
JIANYUN ZHENG ${ }^{1}$, JIANXIN YU ${ }^{2}$, MIN YANG $^{3}$ and LI TANG $^{3}$ \\ ${ }^{1}$ Department of Pathology, The First Affiliated Hospital of Xi'an Medical University, \\ School of General Medicine, Xi'an Medical University, Xi'an, Shaanxi 710077; ${ }^{2}$ Department of Laboratory, \\ Central Hospital of Shanxian County, Heze, Shandong 274399; ${ }^{3}$ Department of Gynaecology and Obstetrics, \\ Puyang Oil Field General Hospital, Puyang, Henan 457001, P.R. China
}

Received July 17, 2018; Accepted March 26, 2019

DOI: $10.3892 / \mathrm{etm} .2019 .7754$

\begin{abstract}
Cervical cancer (CC) is the second most common malignant cancer among women. Gefitinib was one of the first-generation epidermal growth factor receptor-tyrosine kinase inhibitors in clinical trials. However, the underlying mechanism of gefitinib in regulating $\mathrm{CC}$ progression remains unknown. In the current study, two CC cell lines, HeLa and Siha, were used to investigate the effects of gefitinib. Cell counting kit-8 assays demonstrated that treatment with gefitinib exerted strong cytotoxicity in HeLa and Siha cells. Flow cytometry was used to examine cell cycle progression and apoptosis. Treatment with gefitinib enhanced the number of cells in the $G_{0} / G_{1}$ phase and increased apoptosis in HeLa and Siha cells. Furthermore, treatment with gefitinib decreased the protein expression level of $\mathrm{Bcl}-2$ and increased the protein expression level of Bax. Taken together, these results suggest that gefitinib may suppress CC cell proliferation and induce cell cycle arrest and apoptosis. The current study also demonstrated that treatment with gefitinib suppressed epithelial-mesenchymal transition (EMT) as the expression level of the epithelial marker, E-cadherin was increased, while the expression level of the mesenchymal marker, vimentin was decreased. The current study demonstrated that treatment with gefitinib decreased the protein expression levels of phosphorylated-GSK $3 \beta$ and $\beta$-catenin, which suggests that gefitinib may be a potential novel therapeutic strategy in CC by suppressing the Wnt/ $\beta$-catenin signaling pathway and EMT to inhibit tumor metastasis in CC cells. In conclusion, gefitinib may suppress the EMT process during cell invasion
\end{abstract}

Correspondence to: Dr Min Yang or Dr Li Tang, Department of Gynaecology and Obstetrics, Puyang Oil Field General Hospital, 341 Daqing Road, Puyang, Henan 457001, P.R. China

E-mail: zhongnanshan2018@yeah.net

E-mail: smd167725@yeah.net

Key words: cervical cancer, gefitinib, Wnt/ $\beta$-catenin signaling, epithelial-mesenchymal transition and induce cell apoptosis and cell cycle arrest via inhibition of the $\mathrm{Wnt} / \beta$-catenin signaling pathway.

\section{Introduction}

Cervical cancer (CC) is the second most common malignant cancer among women (1). CC is characterized by high incidence and recurrence rates, as well as high resistance to systemic therapies (2). Therefore, prognosis for patients with $\mathrm{CC}$ remains relatively poor. Increasing evidence has demonstrated that the human papillomavirus (HPV) is correlated with the development of a high-grade precursor lesions and invasion in CC (3). HPV can infect epithelial cells, which remain active in cell-cycle progression and no longer undergo apoptosis (4).

Studies have demonstrated that the Wnt signaling pathway serves a key role in cell differentiation, proliferation, migration and polarity $(5,6)$. In addition, the Wnt signaling pathway serves a key role in maintaining protein stability, subcellular localization and transcriptional activity (7). In the progression of tumors, the Wnt/ $\beta$-catenin signaling pathway is an evolutionarily conserved and versatile pathway (8). Aberrant activation of the $\mathrm{Wnt} / \beta$-catenin pathway can lead to abnormal accumulation of $\beta$-catenin in the nucleus, which accelerates the epithelial-mesenchymal transition (EMT) process $(9,10)$. It is therefore important to maintain appropriate Wnt signaling.

Gefitinib was one of the first-generation epidermal growth factor receptor-tyrosine kinase inhibitors in clinical trials, and is now widely used for the treatment of several types of cancer $(11,12)$. An increasing number of studies have been designed to identify the efficacy and toxicity of gefitinib $(13,14)$. However, the underlying mechanism of gefitinib in regulating $\mathrm{CC}$ progression remains unknown. Therefore, the aim of the current study was to investigate the specific role and underlying mechanism of gefitinib in CC using human CC cell lines.

\section{Materials and methods}

Cell culture. Human cervical cancer cell lines HeLa and Siha were obtained from the Institute of Life Sciences Cell Resource Center (Shanghai, China). HeLa and Siha cells were cultured in 
minimal essential medium (MEM, HyClone; GE Healthcare Life Sciences) supplemented with 10\% fetal bovine serum (HyClone; GE Healthcare Life Sciences), penicillin (100 U/ml)-streptomycin (100 U/ml) liquid (Thermo Fisher Scientific, Inc.) and $0.25 \mu \mathrm{g} / \mathrm{ml}$ amphotericin B (Ameresco, Inc.). Cells were cultured at $37^{\circ} \mathrm{C}$ in a humidified incubator containing $5 \% \mathrm{CO}_{2}$.

Cell proliferation assay. HeLa and Siha cell proliferation was examined using the cell counting kit-8 (CCK-8; Dojindo Molecular Technologies, Inc.), according to the manufacturer's protocol. Exponentially growing cells were seeded into 96-well plates at a density of $5 \times 10^{3}$ cells/well in a final volume of $100 \mu \mathrm{l}$ MEM and cultured under normal conditions for $24 \mathrm{~h}$ at $37^{\circ} \mathrm{C}$ in a $5 \% \mathrm{CO}_{2}$-humidified incubator. Subsequently, different concentrations $(0.3125,0.625,1.25,2.5,5,10,20,40$ and $80 \mu \mathrm{mol} / \mathrm{l}$ ) of gefitinib (cat. no. SML1657; Sigma-Aldrich; Merck KGaA; Darmstadt, Germany) were added to each well, with DMSO used as the vehicle control. After $48 \mathrm{~h}, 10 \mu \mathrm{l}$ CCK-8 reagent was added to each well and incubated for $1 \mathrm{~h}$ at $37^{\circ} \mathrm{C}$. Cell proliferation was calculated by measuring the absorbance at a wavelength of $450 \mathrm{~nm}$ using a microplate reader (Bio-Rad Laboratories, Inc.). Growth inhibition was calculated as a percentage of the untreated controls. All experiments were performed in triplicate and the data were expressed as the mean value \pm standard deviation of five wells per treatment. For each cell line, the half maximal inhibitory concentration $\left(\mathrm{IC}_{50}\right)$ was determined using the four-parameter logistic model.

Cell cycle analysis. HeLa and Siha cells were seeded into six-well plates at the density of $10^{6}$ cells/well. Following treatment with $10 \mu \mathrm{mol} / 1$ of gefitinib for $48 \mathrm{~h}$, cells were harvested using trypsin without EDTA, washed three times with ice-cold PBS and fixed with $70 \%$ ethanol overnight at $4^{\circ} \mathrm{C}$. Cells were subsequently stained $25 \mu \mathrm{l}$ propidium iodide with $10 \mu \mathrm{l}$ RNase A at $37^{\circ} \mathrm{C}$ for $30 \mathrm{~min}$ in dark using the Cell cycle and apoptosis analysis kit (cat. no. C1052; Beyotime Institute of Biotechnology, Haimen, China). Cell cycle analysis was performed using a BD FACSCalibur system and CellQuest pro software (version 2.0; BD Biosciences, Franklin Lakes, NJ, USA). All experiments were performed in triplicate.

Flow cytometry evaluation of apoptosis. Cell apoptosis was examined using the Annexin V-fluorescein isothiocyanate (FITC)/propidium iodide (PI) Apoptosis Detection kit (BD Biosciences). Following treatment with $10 \mu \mathrm{mol} / 1$ of gefitinib for $48 \mathrm{~h}$, cells were harvested using trypsin without EDTA and washed three times with ice-cold PBS. Cells were subsequently suspended at $1 \times 10^{4}$ cells/ml in Annexin V-Binding buffer and incubated with $5 \mu \mathrm{l}$ Annexin V-FITC for $15 \mathrm{~min}$ at $37^{\circ} \mathrm{C}$ in dark, followed by staining with $5 \mu \mathrm{PI}$. Apoptotic cells were immediately analyzed using a BD FACSCalibur system and CellQuest pro software. All experiments were performed in triplicate.

Dual-luciferase reporter assay. HeLa and Siha cells were seeded into 24 -well plates at a density of $1 \times 10^{5}$ cells/well and incubated overnight. After $24 \mathrm{~h}$ of culture, cells were co-transfected with 200 ng pTOP-Flash (Promega Corporation, Madison, WI, USA) or pFOP-Flash reporter plasmids (Promega Corporation) and $200 \mathrm{ng} \beta$-galactosidase $(\beta$-gal) using Lipofectamine ${ }^{\circledR} 2000$ (Invitrogen; Thermo Fisher Scientific, Inc.) to monitor for transfection efficiency. After transfection for $48 \mathrm{~h}$, the activity was measured. The TCF-responsive TOP-Flash reporter contains three TCF binding sites, and the corresponding FOP-Flash contains three mutated TCF sites (15). Cells were treated with $10 \mu \mathrm{mol} / \mathrm{l}$ gefitinib for $4 \mathrm{~h}$ at $37^{\circ} \mathrm{C}$ and luciferase activity was analyzed using a Dual-Luciferase Reporter assay system (Promega Corporation), according to the manufacturer's protocol. Luciferase activity was normalized for $\beta$-gal activity. The experiment was performed in triplicate.

Western blot analysis. Total cellular protein was extracted from cells on ice for $15 \mathrm{~min}$ using radioimmunoprecipitation assay buffer (Beyotime Institute of Biotechnology) supplemented with fresh proteinase inhibitor cocktail and phosphatase inhibitor (Sigma-Aldrich; Merck KGaA). Samples were centrifuged at $11,000 \mathrm{x} \mathrm{g}$ for $20 \mathrm{~min}$ at $4^{\circ} \mathrm{C}$. Total protein was quantified using a bicinchoninic acid assay (Sigma-Aldrich; Merck $\mathrm{KGaA}$ ) and $20 \mu \mathrm{g}$ protein/well was separated via SDS-PAGE on a $10 \%$ gel. The fractionated proteins were transferred onto polyvinylidene difluoride membranes (EMD Millipore) and blocked for $1 \mathrm{~h}$ at room temperature with 5\% non-fat skimmed milk. The membranes were incubated with primary antibodies, including E-cadherin (1:1,000; cat. no. ab1416), Vimentin (1:1,000; cat. no. ab8978), GSK3 $\beta$ (1:1,000; cat. no. ab93926), p-GSK3 $\beta$ (1:1,000; cat. no. ab131097), $\beta$-catenin $(1: 1,000$; cat. no. ab32572) and GAPDH (1:5,000; cat. no. ab181602) overnight at $4^{\circ} \mathrm{C}$ (all from Abcam, Cambridge, UK). Following primary antibody incubation, membranes were incubated with horseradish peroxidase-conjugated secondary antibody, horseradish peroxidase-conjugated goat anti-rabbit IgG (1:5,000; cat. no. ZB-2306; OriGne Technologies, Inc.), for $1 \mathrm{~h}$ at room temperature. Protein bands were visualized using a Western Lightning ${ }^{\circledR}$ Chemiluminescence Reagent Plus according to the manufacturer's protocol (cat. no. NEL105001EA; PerkinElmer, Inc., Waltham, MA, USA). Protein expression was quantified using ImageJ (version 1.8.0; National Institutes of Health, Bethesda, MD, USA).

Immunofluorescence. HeLa and Siha cells were cultured in a six-well plate with glass coverslips and following treatment with $10 \mu \mathrm{M}$ gefitinib at $37^{\circ} \mathrm{C}$ for $48 \mathrm{~h}$, cells were fixed with $4 \%$ paraformaldehyde for $30 \mathrm{~min}$ at room temperature. Cells were washed three times with PBS for $5 \mathrm{~min}$ and blocked for $2 \mathrm{~h}$ at room temperature with $8 \%$ bovine serum albumin (BSA; Sigma-Aldrich; Merck KGaA). Subsequently, cells were incubated with primary antibodies against E-cadherin (1:50; cat. no. ab1416) and vimentin (1:50; cat. no. ab92547; both Abcam) in a humidified chamber overnight at $4{ }^{\circ} \mathrm{C}$. Cells were washed three times with PBS. Following primary incubation, cells were incubated with tetramethylrhodamine-conjugated anti-rabbit IgG (1:500; cat. no. ZDR5209; OriGene Technologies, Inc.) at room temperature for $30 \mathrm{~min}$. Cell nuclei were counterstained with DAPI (1:1,000; cat. no. C0060; Beijing Solarbio Science \& Technology Co., Ltd.) for $20 \mathrm{~min}$ at room temperature. Cells were subsequently washed three times with PBS in the dark and the coverslips were mounted with mounting medium at room temperature for $\sim 1 \mathrm{~h}$ in the dark. Fluorescence intensity was observed under 


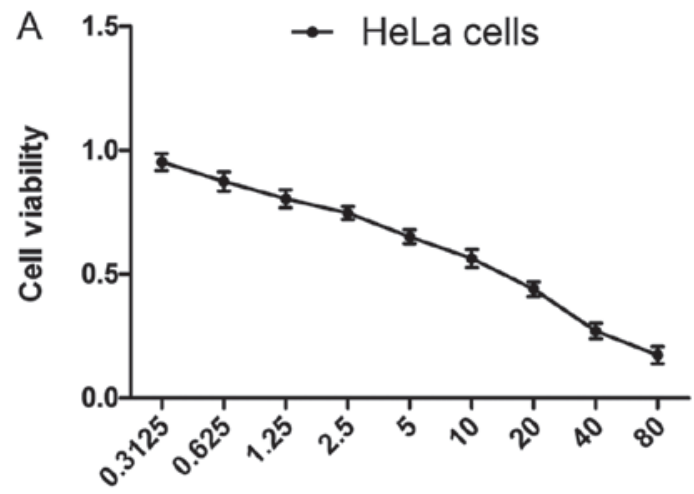

Gefitinib concentration ( $\mu \mathrm{mol} / \mathrm{l})$

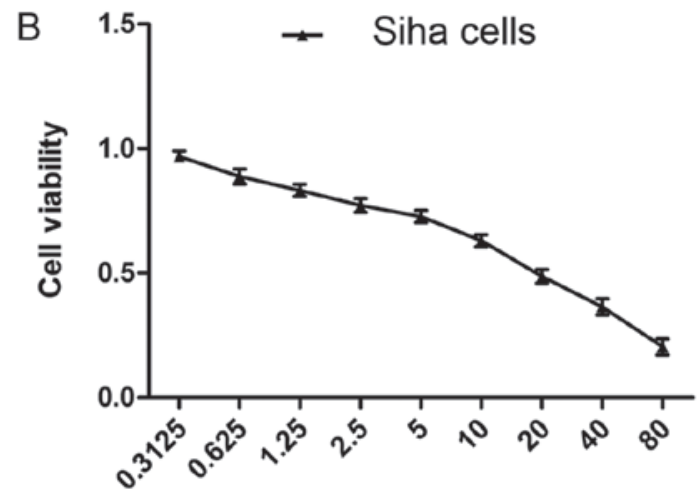

Gefitinib concentration ( $\mu \mathrm{mol} / \mathrm{l})$
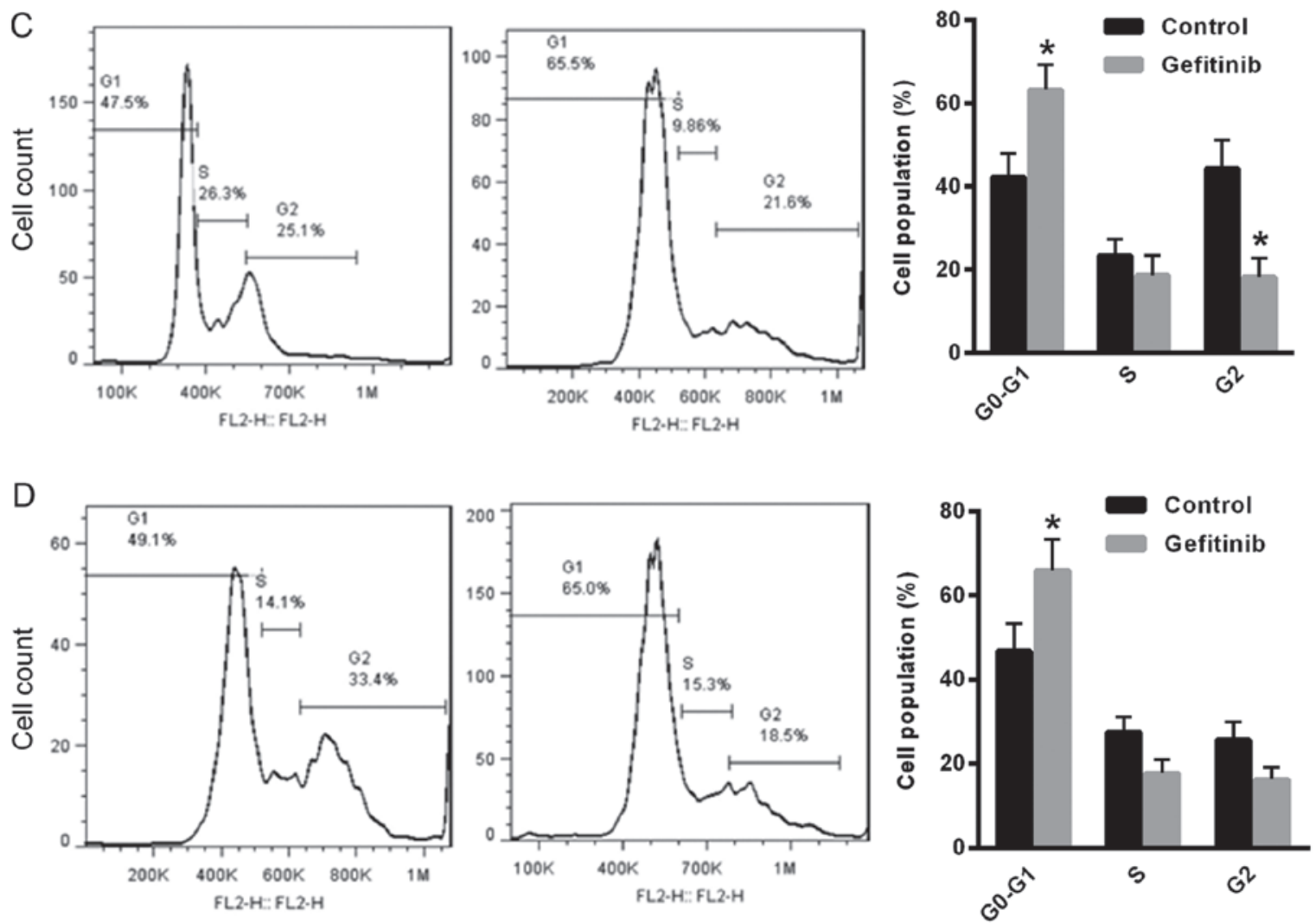

Figure 1. Gefitinib reduces cell proliferation and induces cell cycle arrest in HeLa and Siha cells. Cell proliferation was examined by CCK-8 assay in (A) HeLa and (B) Siha cells following treatment with gefitinib. Cell cycle analysis was determined by flow cytometry in (C) HeLa and (D) Siha cells following treatment with gefitinib. ${ }^{*} \mathrm{P}<0.05$ vs. control. CC, cervical cancer; CCK-8, cell counting kit-8.

a fluorescence microscope (magnification, x40; XDS-500D; Shanghai Caikon Optical Instrument Co., Ltd.).

Statistical analysis. Data were presented as the mean \pm standard error of the mean of at least three experiments. All statistical analyses were performed using GraphPad Prism software (version 5.0; GraphPad Software, Inc.). All experimental data were analyzed using the unpaired Student's t-test or one-way analysis of variance followed by Tukey's post hoc test. $\mathrm{P}<0.05$ was considered to indicate a statistically significant difference.

\section{Results}

Gefitinib reduces proliferation and induces cell cycle arrest in CC cells. To determine the effect of gefitinib on CC cell growth, cell proliferation was examined in HeLa and Siha cells following treatment with gefitinib. Treatment with gefitinib exhibited strong cytotoxicity in HeLa cells [ $\mathrm{IC}_{50}$, $16.19 \pm 0.26 \mu \mathrm{mol} / 1$; $95 \%$ confidence interval (CI): 1.077-1.341; Fig. 1A] and Siha cells $\left(\mathrm{IC}_{50}, 11.87 \pm 0.21 \mu \mathrm{mol} / 1,95 \% \mathrm{CI}\right.$ : 1.003-1.146; Fig. 1B) compared with the control. Furthermore, the effect of gefitinib on cell cycle distribution was examined in HeLa and Siha cells. The number of cells in the $\mathrm{G}_{0} / \mathrm{G}_{1}$ phase was significantly increased in Hela and Siha cells following treatment with gefitinib compared with the control (Fig. 1C and D), which suggests that gefitinib may induce CC cell cycle arrest in the $G_{0} / G_{1}$ phase.

Gefitinib induces apoptosis in CC cells. To examine the effect of gefitinib on CC cell apoptosis, apoptosis was examined 
A HeLa

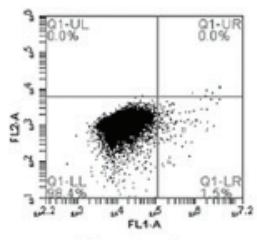

Control

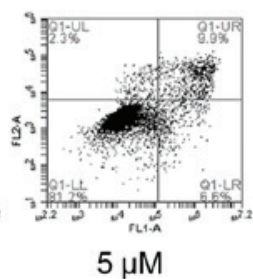

$5 \mu \mathrm{M}$

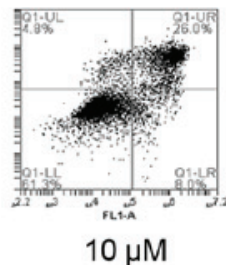

$10 \mu \mathrm{M}$

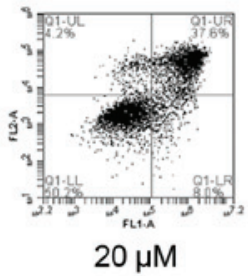

$20 \mu \mathrm{M}$

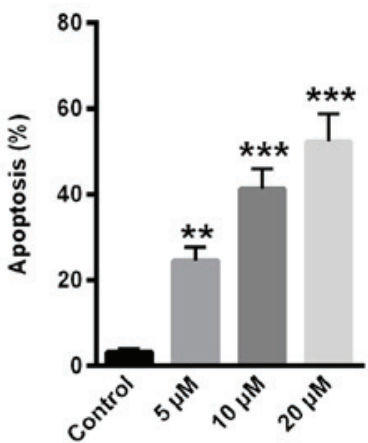

B $\mathrm{SiHa}$

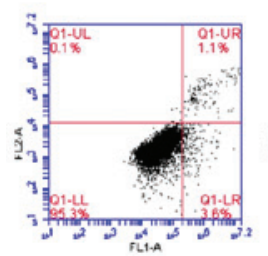

Control

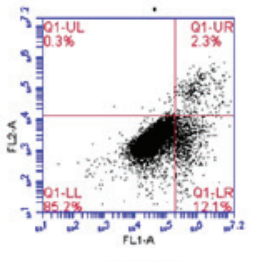

$5 \mu \mathrm{M}$

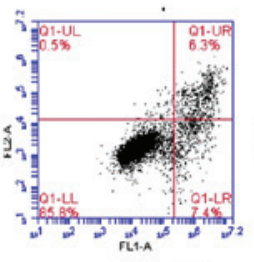

$10 \mu \mathrm{M}$
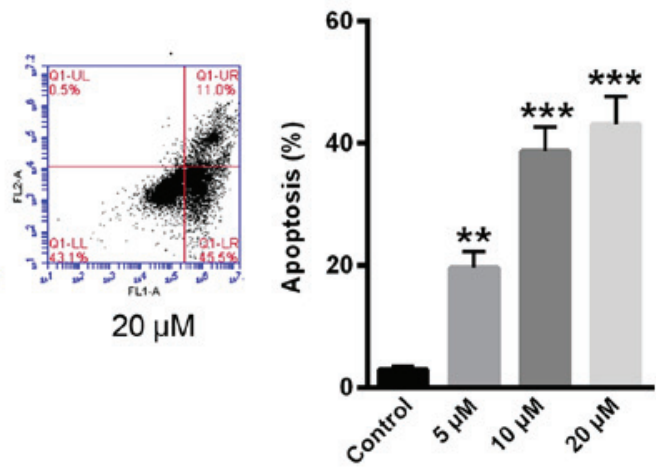

C
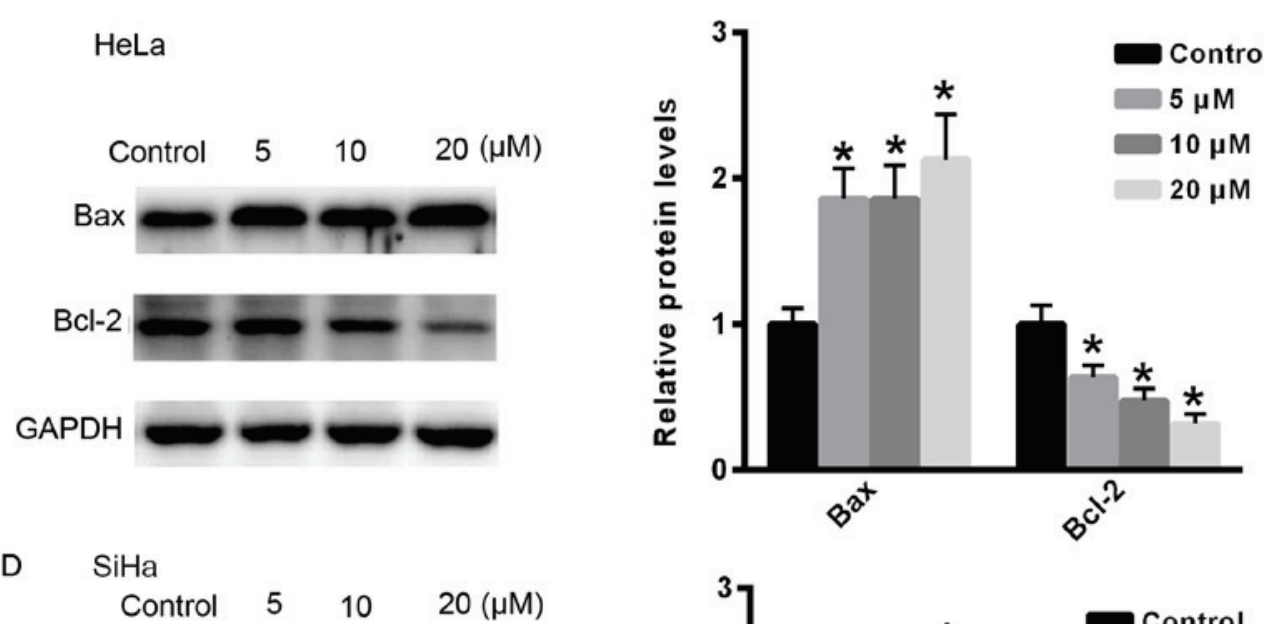

Bax
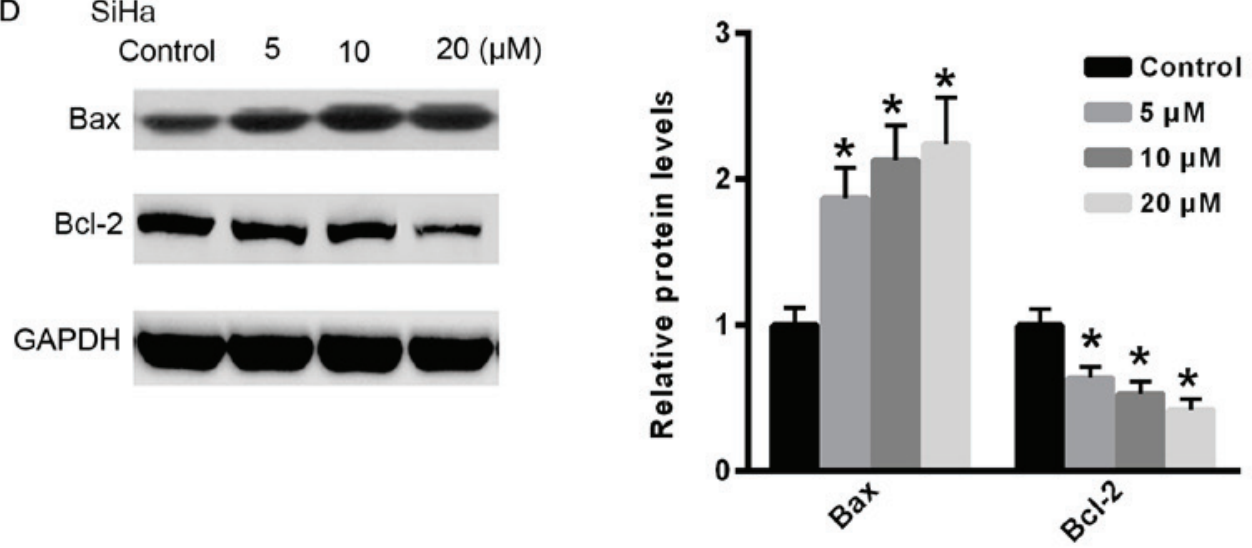

Figure 2. Gefitinib induces apoptosis in HeLa and Siha cells. Cell apoptosis was examined by flow cytometry in (A) HeLa and (B) Siha cells following treatment with gefitinib. The relative protein expression levels of Bcl-2 and Bax were determined by western blot analysis in (C) HeLa and (D) Siha cells following treatment with gefitinib. ${ }^{*} \mathrm{P}<0.05,{ }^{* *} \mathrm{P}<0.01$ and ${ }^{* * *} \mathrm{P}<0.001$ vs. control. Con, control.

in HeLa and Siha cells following treatment with gefitinib. Treatment with gefitinib significantly induced apoptosis in CC cells in a dose-dependent manner compared with the control (Fig. 2A and B). Furthermore, the relative protein expression levels of apoptosis-related proteins, Bax and $\mathrm{Bcl}-2$, were examined. Following treatment with gefitinib, the relative protein expression level of $\mathrm{Bcl}-2$ was significantly reduced, whereas the protein expression level of Bax was significantly increased in HeLa and Siha cells compared with the control (Fig. 2C and D). 
A

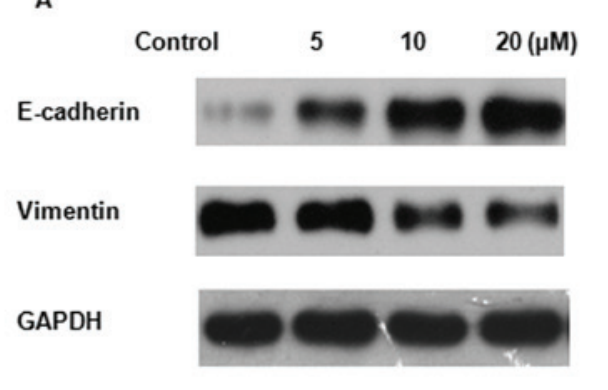

B
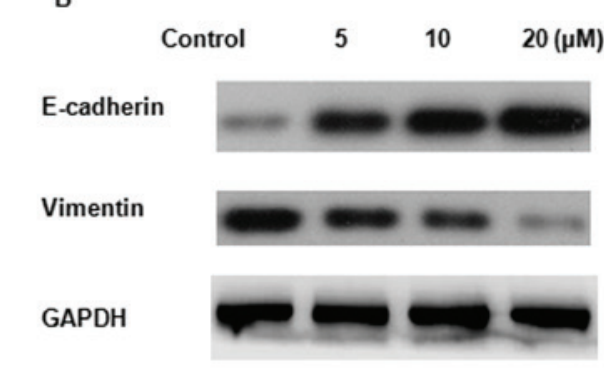

C

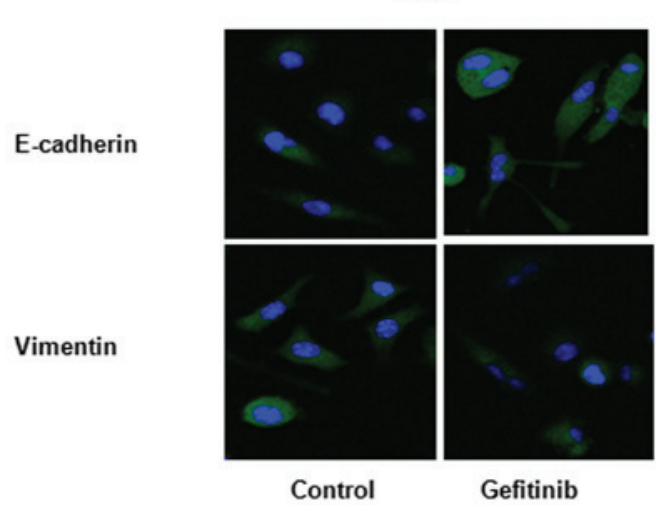

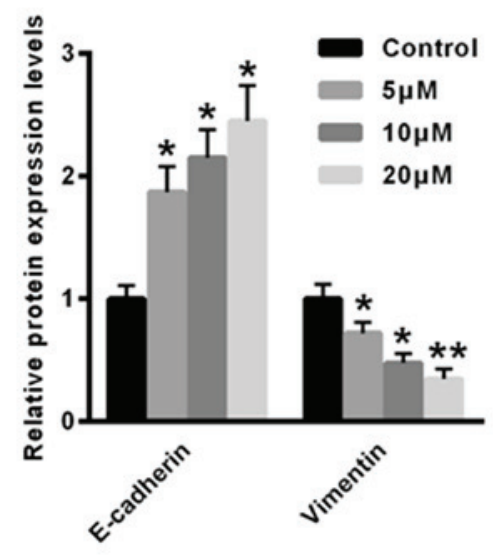

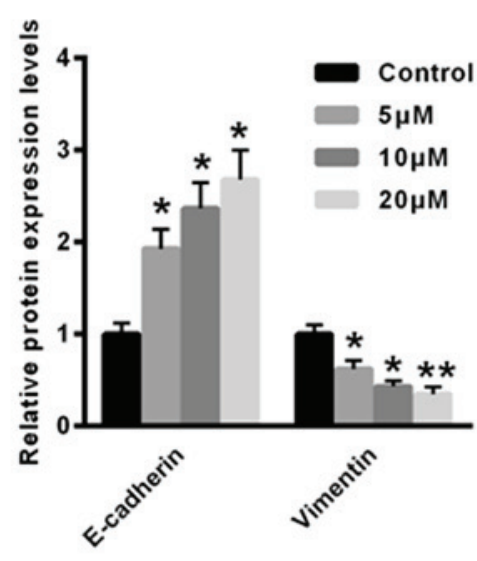

D

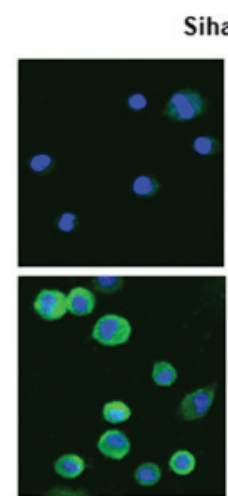

Control

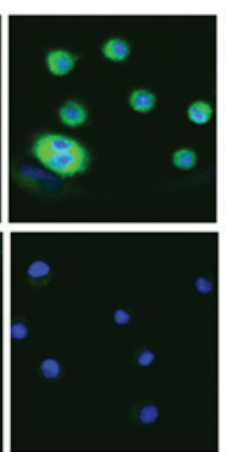

Gefitinib

Figure 3. Gefitinib suppresses EMT in HeLa and Siha cells. The relative protein expression levels of E-cadherin and vimentin were determined by western blot analysis in (A) HeLa and (B) Siha cells following treatment with gefitinib. E-cadherin and vimentin immunostaining in (C) HeLa and (D) Siha cells following treatment with $20 \mu \mathrm{M}$ gefitinib (magnification, $\mathrm{x} 40$ ). ${ }^{*} \mathrm{P}<0.05$ and ${ }^{* *} \mathrm{P}<0.01$ vs. control. EMT, epithelial-mesenchymal transition.

Gefitinib suppresses EMT in CC cells. EMT is a key regulator of CC progression (16). Therefore, the expression levels of E-cadherin, an epithelial cell marker, and vimentin, a mesenchymal cell marker were examined in HeLa and Siha cells following treatment with gefitinib. Following treatment with gefitinib, the protein expression level of E-cadherin was significantly increased, whereas the protein expression level of vimentin was significantly reduced in HeLa and Siha cells compared with the control (Fig. 3A and B). Immunofluorescence demonstrated increased E-cadherin expression and reduced vimentin expression in HeLa and Siha cells following treatment with gefitinib compared with the control (Fig. 3C and D. These results suggest that gefitinib may suppress the EMT process in CC cells.
Gefitinib suppresses EMT via the Wnt/ $\beta$-catenin signaling pathway in $C C$ cells. The $\mathrm{Wnt} / \beta$-catenin signaling pathway is a major contributor to CC tumorigenesis (17). GSK3 $\beta$ is a known negative regulator of $\beta$-catenin. Therefore, the phosphorylation of GSK $3 \beta$ at Ser9, an indicator of the activation state of GSK3 $\beta$, was examined by western blot analysis in HeLa and Siha cells following treatment with gefitinib. The protein expression levels of $\mathrm{p}-\mathrm{GSK} 3 \beta$ and $\beta$-catenin and were significantly reduced in HeLa and Siha cells following treatment with gefitinib compared with the control (Fig. 4A and B). Furthermore, luciferase reporter assays demonstrated that activation of the $\mathrm{Wnt} / \beta$-catenin pathway was significantly suppressed following treatment with gefitinib in HeLa and 
A

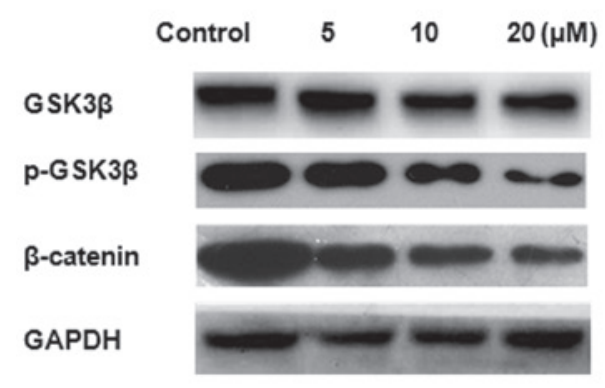

B

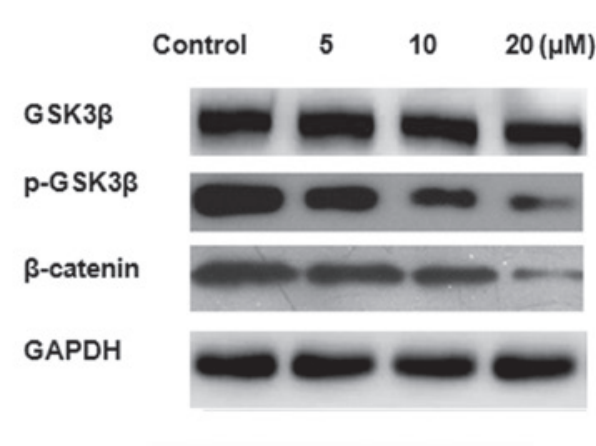

C

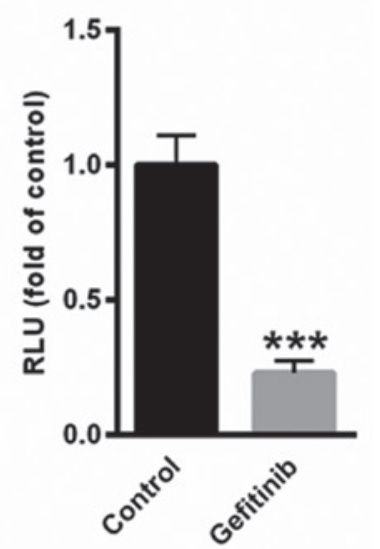

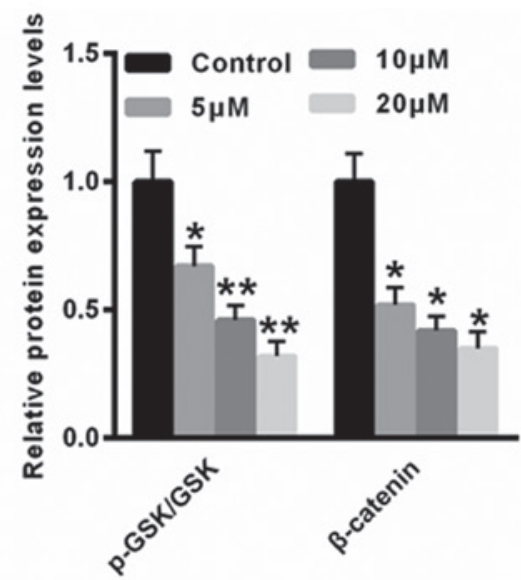

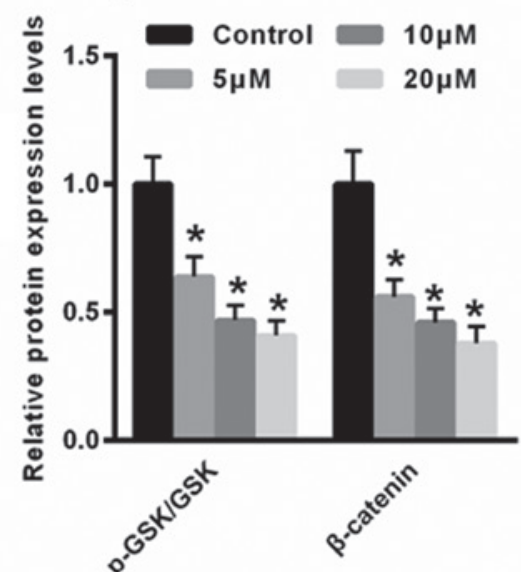

D

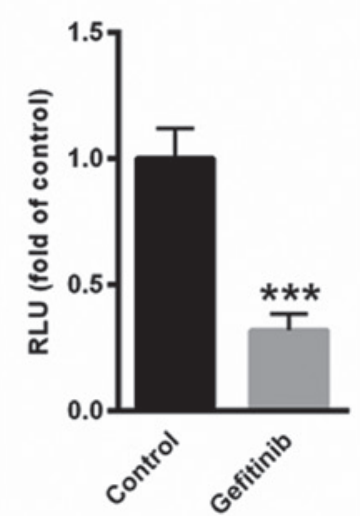

Figure 4. Gefitinib suppresses EMT via the Wnt/ $\beta$-catenin signaling pathway in HeLa and Siha cells. The relative protein expression levels of GSK3 $\beta$, p-GSK $3 \beta$ and $\beta$-catenin were determined by western blot analysis in (A) HeLa and (B) Siha cells following treatment with gefitinib. The activation of the Wnt/ $\beta$-catenin pathway was determined by the luciferase reporter assay in (C) HeLa and (D) Siha cells following treatment with gefitinib. ${ }^{*} \mathrm{P}<0.05$, ${ }^{* *} \mathrm{P}<0.01$ and ${ }^{* * * *} \mathrm{P}<0.001$ vs. control. EMT, epithelial-mesenchymal transition; p-, phosphorylated.

Siha cells compared with the control (Fig. 4C and D). Taken together, these results suggest that gefitinib may inactivate the Wnt/ $\beta$-catenin signaling pathway to inhibit EMT in CC cells.

\section{Discussion}

In recent years, significant advances have been made in the diagnosis and treatment of $\mathrm{CC}$, however, the overall 5-year survival rate remains poor (18). Currently, the most common treatment strategy for CC includes surgery with platinum-based chemotherapy (19). Unfortunately, patients with advanced CC relapse after primary treatment, and the majority of patients succumb to recurrence and metastasis (20). It is therefore necessary to identify novel therapeutic strategies for CC.

The in vitro and in vivo antitumor activity of gefitinib has been reported in several types of human cancer, including head and neck, colorectal, breast and lung cancer (21-23). However, the effect of gefitinib in CC remains unknown. In the current study, two CC cell lines, HeLa and Siha, were used to investigate the effects of gefitinib. CCK- 8 assays demonstrated that gefitinib exerted strong cytotoxicity in HeLa and Siha cells. Flow cytometry was performed to examine cell cycle progression and apoptosis in $\mathrm{CC}$ following treatment with gefitinib. The current study demonstrated that treatment 
with gefitinib enhanced the number of cells in the $G_{0} / G_{1}$ phase and increased apoptosis in HeLa and Siha cells. Furthermore, treatment with gefitinib reduced the protein expression level of Bcl-2, and enhanced the protein expression level of Bax. Taken together, these results suggest that gefitinib may suppress $\mathrm{CC}$ cell proliferation and induce cell cycle arrest and apoptosis.

To further investigate the underlying mechanism of gefitinib in regulating $\mathrm{CC}$ progression, the EMT process was examined in $\mathrm{CC}$ cells following treatment with gefitinib. In the progression of $\mathrm{CC}$, EMT is a key regulator that promotes cancer cell proliferation and invasion (4). The current study demonstrated that treatment with gefitinib suppressed the EMT process by increasing the expression level of the epithelial marker, E-cadherin, and decreasing the expression level of the mesenchymal marker, vimentin. These results suggest that gefitinib may suppress the EMT process in CC.

The canonical Wnt/ $\beta$-catenin signaling pathway serves an important role in EMT $(25,26)$. Abnormal activation of Wnt/ $\beta$-catenin signaling is reported to increase cancer cell proliferation, survival, differentiation and EMT $(27,28)$. The current study examined the potential association between gefitinib and the $\mathrm{Wnt} / \beta$-catenin signaling pathway in $\mathrm{CC}$ cells. The current study demonstrated that treatment with gefitinib decreased the protein expression levels of $p$-GSK3 $\beta$ and $\beta$-catenin, which suggests that gefitinib may be a potential novel therapeutic strategy in $\mathrm{CC}$ by suppressing the Wnt/ $\beta$-catenin signaling pathway and EMT to inhibit tumor metastasis in CC cells.

In conclusion, the current study demonstrated that gefitinib may suppress EMT during cell invasion and induce apoptosis and cell cycle arrest by inhibiting the Wnt/ $\beta$-catenin signaling pathway.

\section{Acknowledgements}

Not applicable.

\section{Funding}

The current study was supported by a grant from Puyang Oil Field General Hospital (grant no. 20160783).

\section{Availability of data and materials}

The datasets used and/or analyzed during the current study are available from the corresponding author upon reasonable request.

\section{Authors' contributions}

JZ performed the experiments and revised the manuscript for important intellectual content. JY performed the cell proliferation experiments and was involved in drafting the manuscript. MY and LT designed the experiments, analyzed the data and gave final approval of the version to be published. All authors read and approved the final manuscript.

\section{Ethics approval and consent to participate}

Not applicable.

\section{Patient consent for publication}

Not applicable.

\section{Competing interests}

The authors declare that they have no competing interests.

\section{References}

1. Zhang R, Lu H, Lyu YY, Yang XM, Zhu LY, Yang GD, Jiang PC, Re Y, Song WW, Wang JH, et al: E6/E7-P53-POU2F1-CTHRC1 axis promotes cervical cancer metastasis and activates Wnt/PCP pathway. Sci Rep 7: 44744, 2017.

2. Bahrami A, Hasanzadeh M, ShahidSales S, Yousefi Z, Kadkhodayan S, Farazestanian M, Joudi Mashhad M, Gharib M, Mahdi Hassanian S and Avan A: Clinical significance and prognosis value of Wnt signaling pathway in cervical cancer. J Cell Biochem 118: 3028-3033, 2017.

3. Chung MT, Lai HC, Sytwu HK, Yan MD, Shih YL, Chang CC, Yu MH, Liu HS, Chu DW and Lin YW: SFRP1 and SFRP2 suppress the transformation and invasion abilities of cervical cancer cells through Wnt signal pathway. Gynecol Oncol 112: 646-653, 2009

4. Cui N, Yang WT and Zheng PS: Slug inhibits the proliferation and tumor formation of human cervical cancer cells by upregulating the $\mathrm{p} 21 / \mathrm{p} 27$ proteins and down-regulating the activity of the Wnt/ $\beta$-catenin signaling pathway via the trans-suppression Akt1/p-Akt1 expression. Oncotarget 7: 26152-26167, 2016.

5. Hua F, Liu S, Zhu L, Ma N, Jiang S and Yang J: Highly expressed long non-coding RNA NNT-AS1 promotes cell proliferation and invasion through $\mathrm{Wnt} /$ beta-catenin signaling pathway in cervical cancer. Biomed Pharmacother 92: 1128-1134, 2017.

6. Kloth JN, Fleuren GJ, Oosting J, de Menezes RX, Eilers PH, Kenter GG and Gorter A: Substantial changes in gene expression of Wnt, MAPK and TNFalpha pathways induced by TGF-beta1 in cervical cancer cell lines. Carcinogenesis 26: 1493-1502, 2005.

7. Kwan HT, Chan DW, Cai PC, Mak CS, Yung MM, Leung TH, Wong OG, Cheung AN and Ngan HY: AMPK activators suppress cervical cancer cell growth through inhibition of DVL3 mediated Wnt/beta-catenin signaling activity. PLoS One 8: e53597, 2013.

8. Lan K, Zhao Y, Fan Y, Ma B, Yang S, Liu Q, Linghu H and Wang $\mathrm{H}$ : Sulfiredoxin may promote cervical cancer metastasis via Wnt/ $\beta$-catenin signaling pathway. Int J Mol Sci 18: E917, 2017.

9. Lee J, Yoon YS and Chung JH: Epigenetic silencing of the WNT antagonist DICKKOPF-1 in cervical cancer cell lines. Gynecol Oncol 109: 270-274, 2008.

10. Li F, Wang T and Tang S: SOX14 promotes proliferation and invasion of cervical cancer cells through $\mathrm{Wnt} / \beta$-catenin pathway. Int J Clin Exp Pathol 8: 1698-1704, 2015.

11. Xu CR, Zhong WZ, Zhou Q, Zhang XC, Yang JJ and Wu YL: Heterogeneity of the resistance to gefitinib treatment in a non-small cell lung cancer patient with active epidermal growth factor receptor mutation. Thorac Cancer 8: 51-53, 2017.

12. Yang RF, Yu B, Zhang RQ, Wang XH, Li C, Wang P, Zhang Y, Han B, Gao XX, Zhang L and Jiang ZM: Bevacizumab and gefitinib enhanced whole-brain radiation therapy for brain metastases due to non-small-cell lung cancer. Braz J Med Biol Res 51: e6073, 2017.

13. Yang XB, Chai XS, Wu WY, Long SQ, Deng H, Pan ZQ, He WF, Zhou YS, Liao GY and Xiao SJ: Gefitinib plus Fuzheng Kang'ai formula () in patients with advanced non-small cell lung cancer with epidermal growth factor receptor mutation: A randomized controlled trial. Chin J Integr Med 24: 734-740, 2018.

14. Yang Z, Hackshaw A, Feng Q, Fu X, Zhang Y, Mao C and Tang J: Comparison of gefitinib, erlotinib and afatinib in non-small cell lung cancer: A meta-analysis. Int J Cancer 140: 2805-2819, 2017.

15. Xu S and Gotlieb AI: Wnt3a/ $\beta$-catenin increases proliferation in heart valve interstitial cells. Cardiovasc Pathol 22: 156-166, 2013.

16. Qureshi R, Arora H and Rizvi MA: EMT in cervical cancer: Its role in tumour progression and response to therapy. Cancer Lett 356: 321-331, 2015.

17. Xu S, Fan Y, Li D, Liu Y and Chen X: Glycoprotein nonmetastatic melanoma protein $B$ accelerates tumorigenesis of cervical cancer in vitro by regulating the Wnt/ $\beta$-catenin pathway. Braz J Med Biol Res 52: e7567, 2018. 
18. Li S, Yang F, Wang M, Cao W and Yang Z: miR-378 functions as an onco-miRNA by targeting the ST7L/Wnt/ $/$-catenin pathway in cervical cancer. Int J Mol Med 40: 1047-1056, 2017.

19. Liu P, Ma S, Liu H, Han H and Wang S: HCFU inhibits cervical cancer cells growth and metastasis by inactivating Wnt/ $\beta$-catenin pathway. J Cell Biochem: Dec 12, 2017 (Epub ahead of print) doi: 10.1002/jcb.26570.

20. Liu XF, Li XY,Zheng PS and Yang WT: DAX1 promotes cervical cancer cell growth and tumorigenicity through activation of Wnt//-catenin pathway via GSK3beta. Cell Death Dis 9: 339, 2018

21. Hartmann S, Neckel N, Seher A, Mutzbauer G, Brands RC, Linz C, Kübler AC and Müller-Richter UD: Erlotinib and gefitinib responsiveness in head and neck cancer cell lines-a comparing analysis with cetuximab. Clin Oral Investig 20: 759-769, 2016.

22. Li Q, Zhang D, Chen X, He L, Li T, Xu X and Li M: Nuclear PKM2 contributes to gefitinib resistance via upregulation of STAT3 activation in colorectal cancer. Sci Rep 5: 16082, 2015.

23. Geng D, Sun D, Zhang L and Zhang W: The therapy of gefitinib towards breast cancer partially through reversing breast cancer biomarker arginine. Afr Health Sci 15: 594-597, 2015.

24. Jiang C, Xu R, Li XX, Wang YY, Liang WQ, Zeng JD, Zhang SS, Xu XY, Yang Y, Zhang MY, et al: p53R2 overexpression in cervical cancer promotes AKT signaling and EMT, and is correlated with tumor progression, metastasis and poor prognosis. Cell Cycle 16: 1673-1682, 2017.
25. Fernando G, Paul F, Laura J, Alejandra AM, Gabriela M and Alberto PL: Is the Wnt/ $\beta$ catenin signalling pathway activated in Seminoma? An immunohistochemical study. J Cancer Res Ther 12: 1075-1079, 2016

26. Yang C, Du W and Yang D: Inhibition of green tea polyphenol EGCG((-)-epigallocatechin-3-gallate) on the proliferation of gastric cancer cells by suppressing canonical wnt/ $\beta$-catenin signalling pathway. Int J Food Sci Nutr 67: 818-827, 2016.

27. Mittag S, Valenta T, Weiske J, Bloch L, Klingel S, Gradl D, Wetzel F, Chen Y, Petersen I, Basler K and Huber O: A novel role for the tumour suppressor Nitrilasel modulating the Wnt/ $\beta$-catenin signalling pathway. Cell Discov 2: 15039, 2016.

28. van Zuylen WJ, Rawlinson WD and Ford CE: The Wnt pathway: A key network in cell signalling dysregulated by viruses. Rev Med Virol 26: 340-355, 2016. 